\title{
Afectación pulmonar en la tularemia
}

\author{
M.A. FERNÁNDEZ JORGE, L. RAMOS CASADO \\ Unidad de Neumología. Hospital "Río Carrión”. Palencia
}

LUNG MANIFESTATIONS OF TULAREMIA

\begin{abstract}
RESUMEN
Presentamos tres casos de neumonía por Francisella tularensis diagnosticados recientemente y revisamos la enfermedad a través de la literatura. Todos los casos eran adultos con antecedente epidemiológico de contacto con liebres en dos de ellos, y presentaron un cuadro clínicoradiológico compatible con la forma neumónica descrita en la literatura El diagnóstico se realizó mediante serología en dos casos y por hemocultivo en el otro. Todos los pacientes respondieron favorablemente al tratamiento antibiótico instaurado, en dos casos con gentamicina y el tercero con un macrólido. La no existencia de referencias al tema en la búsqueda bibliográfica que hemos realizado mediante Medline y en la base de datos españoles (IME) sugiere que pudiera tratarse de los primeros casos descritos en la literatura española.
\end{abstract}

PALABRAS CLAVE: Francisella tularensis. Neumonía. Tularemia.

\begin{abstract}
We present three cases of pneumonia by Francisella Tularensis recently diagnosed. We also review this disease with the literature. All the studied patients were adults; two of them had epidemiological antecedents because of being in contact with hares. They present a clinicalradiological symptons compatible with the pneumonic case described in the literature. The diagnosis was realized through serology in two cases and hemocultive in the other one. All patients had a positive answer to the antibiotic treatment, two cases with gentamicine and the other one with macrolide. There are not references about the subject in the biblio graphical research we have realized in Medline. We did not find infor mation in the Spanish base (IME), perhaps that these were the first cases found in the Spanish literature.
\end{abstract}

KEY WORDS: Francisella tularensis. Pneumonia. Turalemia.

Fernández Jorge MA, Ramos Casado L. Afectación pulmonar en la tularemia. An Med Interna (Madrid) 2001; 18: 32-34.

\section{INTRODUCCIÓN}

La tularemia es una enfermedad infecciosa que afecta inicialmente a determinados animales y se transmite de forma ocasional al hombre directamente o mediante un artrópodo vector. Es una enfermedad poco frecuente y prácticamente desconocida en nuestro país, no existiendo en la búsqueda por Medline ni en IME referencias bibliográficas españolas en la literatura. La mayoría de los estudios clínicos y epidemiológicos proceden de EE.UU. donde se diagnostican entre 150-300 casos cada año $(0,05-0,15 / 100.000$ habitantes) (1).

Recientemente en la Comunidad de Castilla-León se han detectado varios casos de tularemia con diferentes manifestaciones clínicas, entre las que se encuentra la forma neumónica. Hemos tenido la oportunidad de diagnosticar tres casos de neumonía por Francisella tularensis cuyos datos recogemos en este artículo, así como una revisión de la enfermedad a través de la literatura.

\section{CASOS APORTADOS}

Caso 1: Varón de 74 años de edad, EPOC, hipertenso e intervenido de hernia inguinal y desprendimiento de retina. Acude a urgencias por cuadro de una semana de evolución de síndrome constitucional y fiebre. Como antecedente epidemiológico refiere haber despellejado una liebre diez días antes. En la exploración física destaca a la auscultación pulmonar crepitantes en base derecha. Resto dentro de la normalidad. En la Rx de tórax se observa imagen de aumento de densidad a nivel de LMD con pequeño derrame pleural homolateral. En el test de microaglutinación se obtiene un título para $F$. tularensis de 128. Repetido tres semanas después el título aumenta a 2,048. El paciente fue tratado con gentamicina presentando buena evolución clínica y radiológica, permaneciendo asintomático en la actualidad.

Trabajo aceptado: 10 de Mayo de 1999

Correspondencia: $\mathrm{M}^{\mathrm{a}}$ Angeles Fernández Jorge. c/ Ignacio Serrano 1, Chalet 10. 47008 Valladolid. 
Caso 2: Mujer de 49 años remitida desde otro centro para estudio de masa hiliar derecha. Como antecedentes personales presenta una DM tipo II. Trabaja como ganadera y refiere contacto con liebres. Inicialmente el motivo de consulta fue fiebre y síndrome constitucional. Exploración física dentro de la normalidad En la analítica destaca GPT 152 UI/L y GGT $151 \mathrm{UI} / \mathrm{L}$ con leve hipoxemia al ingreso con $\mathrm{FiO}_{2}$ 0,21 ( $\left.\mathrm{pO}_{2} 56 \mathrm{mmHg}\right)$. En Rx de tórax masa hiliar derecha de aproximadamente $4 \mathrm{~cm}$ con infiltrado a nivel de LSD. Se realizó broncoscopia observándose inflamación en la carina de división entre el segmento anterior y apical del LSD. Test de microaglutinación para Francisella tularensis de 128. Repetido a las tres semanas se alcanza un título superior a 1048. Tras tratamiento empírico con macrólidos, la paciente presentó buena evolución clínica y radiológica.

Caso 3: Varón de 77 años intervenido de hidrocele izquierdo y trabajador en la agricultura que acude a urgencias por comenzar 15 días antes con fiebre y malestar general. Exploración física dentro de la normalidad En la analítica únicamente destaca GPT 73 UI/L y GGT 83 UI/L. En la Rx de tórax se observa imagen de condensación alveolar en campo medio derecho con pinzamiento del seno costofrénico homolateral (Fig. 2). El hemocultivo fue positivo para Francise lla tularensis. El paciente fue tratado de modo empírico con ceftriaxona y gentamicina quedando asintomático.

\section{DISCUSIÓN}

La tularemia está causada por un pequeño coco-bacilo gram negativo denominado Francisella tularensis en honor a Edward Francis que describió su experiencia sobre 800 casos en 1928 y a Tulare (California), lugar donde fue aislado (2).

La transmisión de la enfermedad al hombre suele producirse a través de un artrópodo vector (garrapatas, pulgas, tal vez mosquitos) $(2,3)$ o mediante contacto directo con animales contaminados (liebres, conejos, ardillas) (2-5).

Las manifestaciones clínicas de la enfermedad son variables admitiéndose seis formas clínicas estrechamente relacionadas con la forma de transmisión (6-12) (Tabla I).

La forma pulmonar o neumónica puede presentarse en el contexto de una forma tifoidea o aparecer como única manifestación de la tularemia hasta en el $20 \%$ de los casos $(7,13,14)$. Se produce cuando el microorganismo es inhalado y se disemina posteriormente por vía hematógena. Radiológicamente suele presentar infiltrados lobares o segmentarios,

\section{TABLA I}

\begin{tabular}{|c|c|c|}
\hline \multicolumn{3}{|c|}{ FO RM AS CLÍNICAS DE LA TULAREM IA } \\
\hline & Descripción & Frecuencia \\
\hline Úlcero-glandular & Úlcera cutánea & $45-75 \%$ \\
\hline Glandular & $\begin{array}{l}\text { Adenopatía } \\
\text { Sin lesion cutánea }\end{array}$ & $15-20 \%$ \\
\hline Orofaríngea & $\begin{array}{l}\text { Tonsilitis, Faringitis } \\
\text { Adenopatía regional }\end{array}$ & $4-18 \%$ \\
\hline O culo-glandular & $\begin{array}{l}\text { Conjuntivitis } \\
\text { Adenopatía preauricular }\end{array}$ & $1-4 \%$ \\
\hline Tifoidea & $\begin{array}{l}\text { S. febril, vómito, diarrea } \\
\text { Exantema, } \\
\text { hepatoesplenomegalia }\end{array}$ & $8-18 \%$ \\
\hline Pulmonar & Cuadro pleuropulmonar & $20 \%$ \\
\hline
\end{tabular}

adenopatías hiliares y derrame pleural (15). Se han descrito también formas cavitarias e infiltrados miliares (16). El derrame pleural de la tularemia tiene características de exudado linfocítico y en ocasiones el ADA está elevado (17). El diagnóstico se realiza generalmente por serología. Los anticuerpos se

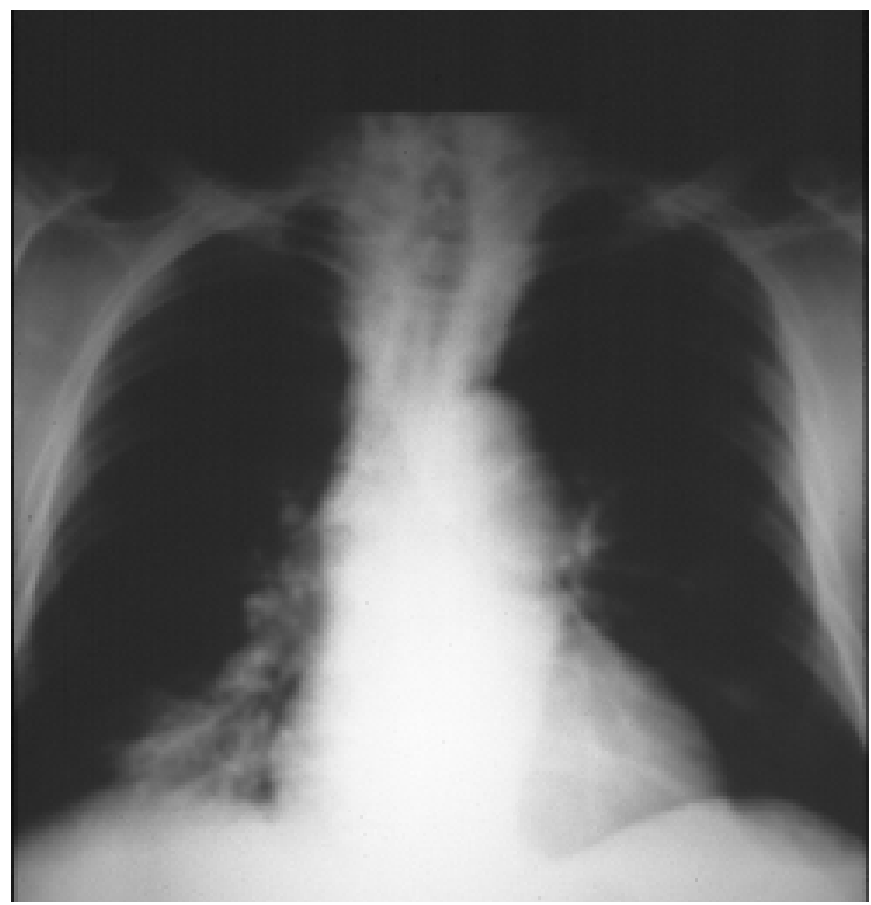

Fig. 1. Caso 1: Infiltrado a nivel de LMD con pequeño derrame pleu ral homolateral.

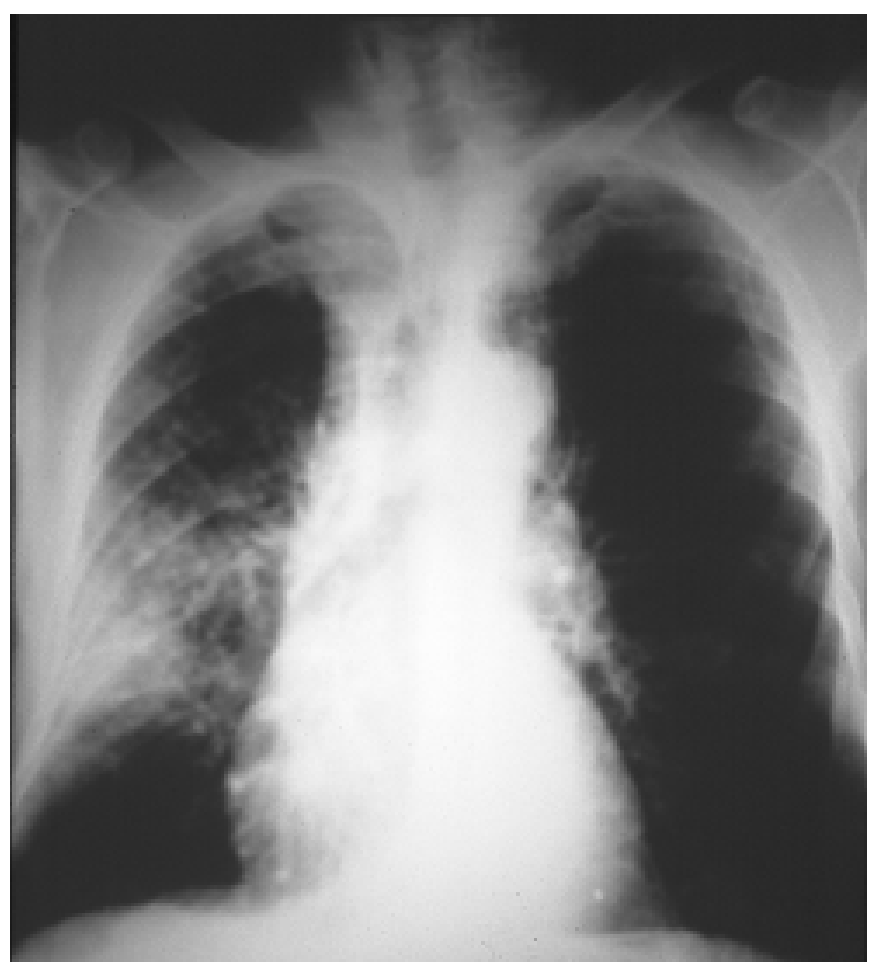

Fig. 2. Caso 3: Imagen de condensación en campo medio derecho con pinzamiento del seno costofrénico homolateral. 
detectan a partir de la $2^{\mathrm{a}}$ semana y pueden permanecer elevados durante años $(6,7)$. Un título inicial igual o superior a 160 sugiere enfermedad pero debe confirmarse repitiendo la determinación en las 4-6 semanas siguientes.

Para el tratamiento se aconseja estreptomicina o gentamicina como alternativa de similar efectividad $(7,18)$ aunque se han descrito buenos resultados con tetraciclinas, cloranfenicol y ciprofloxacino y más dudosos con ceftriaxona e imipenen (18-21).

Los tres casos observados por nosotros corresponden a la forma pulmonar o neumónica descrita en la literatura. Todos ellos presentaron fiebre y síndrome constitucional como síntomas iniciales y en dos de ellos es claro el antecedente epidemiológico de contacto con liebres. Las alteraciones radiológicas que presentaron corresponden también a las descritas como habituales (Fig. 1 y 2).

El diagnóstico se efectuó por serología en dos casos y mediante hemocultivo en el caso tercero. Este hecho es destacado por su rareza ya que es excepcional el aislamiento de Francisella tularensis en hemocultivo sobre todo en Europa $(22,23)$. Estos casos tienen en común su manifestación clínica en forma séptica con afectación pleuropulmonar y rabdomiolisis (22). Nuestros pacientes no presentaron este último aspecto, tan solo detectamos elevación de transaminasas en los casos 2 y 3 como única alteración analítica que también se recoge en la literatura como habitual en la tularemia (2). En cuanto al tratamiento, aunque los tres casos respondieron favorablemente, solo dos recibieron gentamicina ante la sospecha clínica. El caso 2 fue tratado empíricamente con un macrólido, confirmándose el diagnóstico posteriormente cuando el cuadro clínico-radiológico había remitido. No hemos encontrado referencias bibliográficas de la respuesta de Francise lla tularensis a macrólidos salvo un artículo noruego de 1993 (24) donde los macrólidos presentaron un patrón medio de sensibilidad-resistencia frente a un claro patrón de sensibilidad para gentamicina, cloranfenicol, doxicilina y quinolonas y un patrón de resistencia para penicilina, cefalosporinas (cefalexina, cefuroxima ,ceftazidima), aztreonam e imipenem. Concluimos en la conveniencia de incluir la tularemia en el diagnóstico diferencial de los procesos infecciosos en general y los pleuropulmonares en particular, recordando que en estos casos la clínica, radiología y las características del líquido pleural pueden simular una tuberculosis.

Nota: Con posterioridad a la redacción de este manuscrito hemos podido constatar la existencia de varios artículos sobre tularemia, publicados todos ellos en el ${ }^{\circ} 12$ de la Revista Clí nica Española de diciembre de 1998. No obstante, no hemos encontrado en ninguno de ellos descripción de alguna forma pulmonar.

\section{Bibliografía}

1. CDC. Summary of notifiable diseases, United States, MMWR 1991: 40-51.

2. Goddard J. Arthropod transmission of tularemia. Infect Med 1998; 15: 306-8.

3. Weinberg AN. Tularemic Pneumoniae due to Francisella Tularensis. Respiratory Infections: Diagnosis and Mangement. 2a Ed. J. Pennington Raven Press. New York 1988: 422-3.

4. Morner T. The ecology of tularemia. Rev Sci Tech 1992; 11: 1123-30.

5. Taylor JP, Estre GR, Mcchesney TC, Sahalowich FT, Parker RL, Mcfarland LM. Epidemiologic characteristics of human tularemia in the southwest-central states 1981-1987. Am J Epidemiol 1991; 133: 1032-8.

6. CDC. Case definitions for public health surveillance. MMWR 1990; 39: 40.

7. Cross JT. Tularemia in the Umted States. Infect Med 1997; 14: 881-90.

8. Rohrbach BW, Westerman E, Estre GR. Epidemiology and clinical characteristics of tularemia in Oklahoma, 1979 to 1985 . South Med J 1991; 84: 1091-6.

9. Luotonen J, Syrjala H, Jokinen K, Sutinen S, Salminen A. Tularemia in Otolaryngologia practice. An analysis of 127 cases. Arch. Otolaringol. Head New Surg 1986; 112: 77-80.

10. Nordahl SH, Hoel T, Scheel O, Olofsson J. Tularemia: a differential diagnosis in Otorhinolaryngology. J Laringol Otol 1993; 107: 127-9.

11. Syrjala H, Karvonen J, Salminen A. Skin manifestations of tularemia: a study of 88 cases in northern Finlad during 16 years (1967-1983). Acta Derm Venereol 1984; 64: 513-6.

12. Evans M.E., Gregory D. W., Shaffner W., Mcgee Z.A. Tularemia: a 30 year Experience with 88 cases. Medicine (Baltimore) 1985; 64: 251-69.

13. Gill V, Cunha BA. Tularemia pneumonia. Semin Respir Infect 1997; 12: $61-7$.
14. Scofield RH, López EJ, Mcnabb SJ. Tularemia pneumonia in Oklahoma, 1982-1987. J. Okla State Med 1992; 85: 165-70.

15. Rubin SA. Radiographic spectrum of pleuropulmonary tularemia. AJR Am J Roentgeol 1978; 131: 277-81.

16. Kozak AJ, Hall WH, Gerding DN. Cavitary pneumonia associated with Tularemia. Chest 1978; 73: 426-7.

17. Petterson T, Nyberg P, Nordstrom D, Riska H. Similar pleural fluid findings in pleuropulmonary tularemia and tuberculous pleurisy. Chest 1996; 109: 572-5.

18. Enderlin G, Morales L, Jacobs RF, Cross JT. Streptomycin and alternative Agents for the treatment of tularemia: review of the literature. Clin Infect Dis 1994; 19: 42-7.

19. Cross JT, Jacobs RF. Tularemia: Treatment failures with outpatient use of ceftriaxone. Clin Infect Dis 1993; 17: 976-80.

20. Risi GF, Pombo DJ. Relapse of tularemia after aminoglycoside theray: case report and discussion of therapeutic options. Clin Infect Dis 1995; 20: $174-5$.

21. Scheel 0, Reiersen R, Huel T. Treatment of tularemia with ciprofloxacin. Eur J Clin Microbiol Infect Dis 1992; 11: 447-8.

22. Provenza JM, Klotz SA, Penn RL. Isolation of Francisella tularensis from Blood. J Clin Microbiol 1986; 24: 453-5.

23. Hoel T, Scheel 0, Nordahl SH, Sandvik T. Water and airborne Francisella Tularensis isolated from humanblood. Infection 1991; 19: 34850 .

24. Scheel 0, Hoel T, Sandvik T, Berdal BP. Susceptibility pattern of Scandinavian Francisella Tularensis isolates with regard to oral and parenteral antimicrobial agents. APMIS 1993; 101: 33-6. 